\title{
Comparative Genomic Analysis of Bacillus thuringiensis Reveals Molecular Adaptations to Copper Tolerance
}

\author{
Low Yi Yik ${ }^{1}$; Grace Joy Wei Lie Chin ${ }^{1}$; Collin Glen Joseph²; Kenneth Francis Rodrigues ${ }^{1 \#}$
}

\footnotetext{
${ }^{1}$ Biotechnology Research Institute, University Malaysia Sabah, Kota Kinabalu, Sabah, Malaysia.

${ }^{2}$ Faculty of Science and Natural Resources, University Malaysia Sabah, Kota Kinabalu, Sabah, Malaysia. \#Email: kennethr@ums.edu.my
}

\begin{abstract}
Bacillus thuringiensis is a type of Gram positive and rod shaped bacterium that is found in a wide range of habitats. Despite the intensive studies conducted on this bacterium, most of the information available are related to its pathogenic characteristics, with only a limited number of publications mentioning its ability to survive in extreme environments. Recently, a B. thuringiensis MCMY1 strain was successfully isolated from a copper contaminated site in Mamut Copper Mine, Sabah. This study aimed to conduct a comparative genomic analysis by using the genome sequence of MCMY1 strain published in GenBank (PRJNA374601) as a target genome for comparison with other available $B$. thuringiensis genomes at the GenBank. Whole genome alignment, Fragment all-againstall comparison analysis, phylogenetic reconstruction and specific copper genes comparison were applied to all forty-five $B$. thuringiensis genomes to reveal the molecular adaptation to copper tolerance. The comparative results indicated that $B$. thuringiensis MCMY1 strain is closely related to strain Bt407 and strain IS5056. This strain harbors almost all available copper genes annotated from the forty-five B. thuringiensis genomes, except for the gene for Magnesium and cobalt efflux protein (CorC) which plays an indirect role in reducing the oxidative stress that caused by copper and other metal ions. Furthermore, the findings also showed that the Copper resistance gene family, CopABCDZ and its repressor (CsoR) are conserved in almost all sequenced genomes but the presence of the genes for Cytoplasmic copper homeostasis protein (CutC) and $\mathrm{CorC}$ across the sample genomes are highly inconsonant. The variation of these genes across the $B$. thuringiensis genomes suggests that each strain may have adapted to their specific ecological niche. However, further investigations will be need to support this preliminary hypothesis.
\end{abstract}

Keywords: Comparative genomics, Bacillus thuringiensis, Mamut Copper Mine, Copper homeostasis

\section{Introduction}

Copper is an important trace element required by living organisms in a very small amount to sustain the functioning of enzymes. However, excessive amount of copper can be harmful to life, this is because of its semi-soluble property in water. Copper can perform one electron redox chemistry and produces free $\mathrm{Cu}(\mathrm{I})$ radicals that target the enzymes associated with the intermediary metabolism [1]. In nature, copper occurred in rock, soil or sediment systems, due to the disturbance of natural event or human activities such as mining operations, overexposure of copper minerals occurs and this can lead to environmental pollutions and the chain reaction can affect the ecosystem. Some microorganisms such as bacteria are known to have high endurance towards copper pollution and able to thrive in extreme niches which are contaminated by copper [2]. B. thuringiensis is a rod shaped soil bacteria that have also been studied intensively as a candidate of biological pesticide. In early of the $20^{\text {th }}$ century, B. thuringiensis was discovered as a pathogen to silk worm larvae and it was named after a German Town, Thuringia later [3]. After several decades of investigations, the pathogenicity of $B$. thuringiensis was found to be associated with the Crystal protein produced by the bacteria itself. Over the years, several publications had made to prove that $B$. thuringiensis were found to be surviving at environments that are associated with copper pollution, such as acid mine drainage [4], heavy metal polluted wastewater [5] and industrial effluent contaminated soil [6]. However, some publications also addressed this bacteria as a common mesophile that has minimal tolerance to copper [7]. Nevertheless, none of these studies have provided any comparisons in the genetic and molecular aspects. Recently, a Bacillus thuringiensis strain MCMY1 was isolated from the copper contaminated soil 
Table 1. All B. thuringiensis genomes selected for comparison analysis.

\begin{tabular}{|c|c|c|c|}
\hline B. thuringiensis strain & Origins & Size $(\mathrm{Mb})$ & BioProject \\
\hline MCMY1 & Mamut Copper Mine, Sabah, Malaysia & 5.45815 & PRJNA374601 \\
\hline $97-27(1)$ & Human wound, Yugoslavia & 5.31479 & PRJNA10877 \\
\hline $97-27(2)$ & Human tissue, USAMRIID & 5.31269 & PRJNA238078 \\
\hline YBT-1518 & Soil, Jianghan plain, China & 6.67292 & PRJNA63189 \\
\hline Al Hakam & Iraq & 5.31303 & PRJNA18255 \\
\hline BMB171 & Huazhong Agricultural University, China & 5.64305 & PRJNA43631 \\
\hline YBT-020 & Huazhong Agricultural University, China & 5.68238 & PRJNA60447 \\
\hline CT-43 & Huazhong Agricultural University, China & 6.15115 & PRJNA43737 \\
\hline HD-771 & Los Alamos National Laboratory & 6.43837 & PRJNA171845 \\
\hline HD-789 & USDA Agricultural Research Service, Peoria IL & 6.33463 & PRJNA171844 \\
\hline MC28 & Mu Chuan virgin forest, China & 6.69453 & PRJNA167562 \\
\hline Bt407 & Goettingen Genomics Laboratory & 6.13434 & PRJNA176850 \\
\hline HD73 & Centre OILB, Institut Pasteur, France & 5.90857 & PRJNA185468 \\
\hline IS5056 & University of Bialystok & 6.77159 & PRJNA187142 \\
\hline YBT-1520 (1) & Soil, China & 6.58054 & PRJNA181183 \\
\hline YBT-1520 (2) & Huazhong Agricultural University, China & 6.52041 & PRJNA242813 \\
\hline HD-1 & Dead pink bollworm, Texas, USA & 6.76659 & PRJNA181182 \\
\hline HD-29 & Dendrolimus sibericus, Czechoslovakia & 6.74223 & PRJNA261635 \\
\hline HD1011 & India & 6.09337 & PRJNA238081 \\
\hline HD571 & Los Alamos National Laboratory & 5.31218 & PRJNA238070 \\
\hline HD682 & Los Alamos National Laboratory & 5.29139 & PRJNA238078 \\
\hline HD1002 & Sewage, Israel & 6.5727 & PRJNA236049 \\
\hline BGSC-4AA1 & Huazhong Agricultural University, China & 6.1799 & PRJNA271502 \\
\hline YC-10 & Roots of tobacco, China & 6.78414 & PRJNA280152 \\
\hline HS18-1 & Soil, Sichuan, China & 6.40346 & PRJNA288953 \\
\hline HD521 & Soil, USA & 6.19845 & PRJNA263441 \\
\hline YWC2-8 & Soil, Sichuan basin, China & 6.22794 & PRJNA299545 \\
\hline CTC & Soil, Wuhan, China & 5.35293 & PRJNA302721 \\
\hline tolworthi & Saga University & 6.87059 & PRJDB3909 \\
\hline Bt185 & Soil, China & 6.3905 & PRJNA311097 \\
\hline HD12 & Soil, USA & 6.49046 & PRJNA302106 \\
\hline Bc601 & Tianjin, China & 6.11129 & PRJNA317300 \\
\hline BGSC-4C1 & Bombyx mori, Czechoslovakia & 5.81812 & PRJNA317856 \\
\hline MYBT18246 & Caenorhabditis elegans & 6.75249 & PRJNA290307 \\
\hline KNU-07 & Ginseng, South Korea & 6.15274 & PRJNA330597 \\
\hline Bt18247 & Caenorhabditis elegans & 6.1382 & PRJNA227492 \\
\hline L-7601 & Tianjin, China & 6.30377 & PRJNA377597 \\
\hline ATCC $10792(1)$ & South Korea & 6.31628 & PRJNA383629 \\
\hline ATCC $10792(2)$ & Animal tissue, USA & 5.70184 & PRJNA29723 \\
\hline SCG04-02 & Soil, Taiga, China & 5.87824 & PRJNA344831 \\
\hline YGD22-03 & Soil, Woodland, China & 5.67554 & PRJNA359733 \\
\hline BM-BT15426 & Guangzhou, China & 5.24633 & PRJNA381833 \\
\hline $\mathrm{c} 25$ & Soil, Grassland, South Korea & 5.66119 & PRJNA388287 \\
\hline XL6 & Soil, Inner Mongolia, China & 6.44517 & PRJNA244583 \\
\hline ST7 & Soil, Sichuan Basin, China & 6.28612 & PRJNA299545 \\
\hline
\end{tabular}


collected from Mamut Copper Mine, Sabah, Malaysia [8]. The genome of B. thuringiensis MCMY1 was sequenced by using Single Molecular Real Time sequencing (SMRT) approach of Pacific Bioscience and assembled by using SMRT Portal. $B$. thuringiensis strain MCMY1 had shown its ability to withstand a Minimum Inhibitory Concentration (MIC) of $1.7 \mathrm{mM}$ of simulated copper stress in previous experiment [8]. This study is driven by the interest to investigate the evolutionary adaptation of $B$. thuringiensis to copper tolerance by using comparative genomic approach. B. thuringiensis MCMY1 strain was used as the target strain for comparison with other available completed genomes of $B$. thuringiensis in NCBI GenBank database.

\section{Materials and Methods}

The genome of B. thuringiensis strain MCMY1 (PRJNA374601) was used as the target genome for comparison analysis. Other $B$. thuringiensis genomes were selected among those submitted as of May, 2018 in NCBI GenBank genome database and together with a control genome of Escherichia coli strain O157:H7 Sakai. All B. thuringiensis genomes were selected by referring to the genome and assembly report of $B$. thuringiensis under the category of completed genome in the GenBank. The list of all $B$. thuringiensis genomes and their responding strains have been described in Table 1 . The nucleotide sequences of all strains were retrieved in FASTA format from the GenBank genome database. The quality of the assemblies for all genomes were assessed by using QUAST version 4.6.3 [9]. Whole genome alignment and Fragment all-against-all comparison analysis was done by using Gegenees software version 2.2.1 with BLAST+ version 2.7.1 installed in the pipeline [10]. A heat plot for the genomes comparison was generated from Gegenees software and exported in Nexus file format to be used for further phylogenomic analysis. SplitsTree software version 4 [11] was used for computing of phylogenetic tree. All B. thuringiensis genomes selected were uploaded to Rapid Annotations using Subsystems Technology (RAST) version 2.0 for annotations [12]. The summary of all annotations were viewed in the SEED viewer of RAST [13]. All genes annotated in the subsystems of copper homeostasis, copper tolerance, copper uptake, copper transport system and blue copper proteins were retrieved from the database. BLAST Ring Image Generator (BRIG) was used to compare the presence of these annotated copper related genes with a customized multi-FASTA file that contained the annotations information as the reference genome [14].

\section{Result and Discussion}
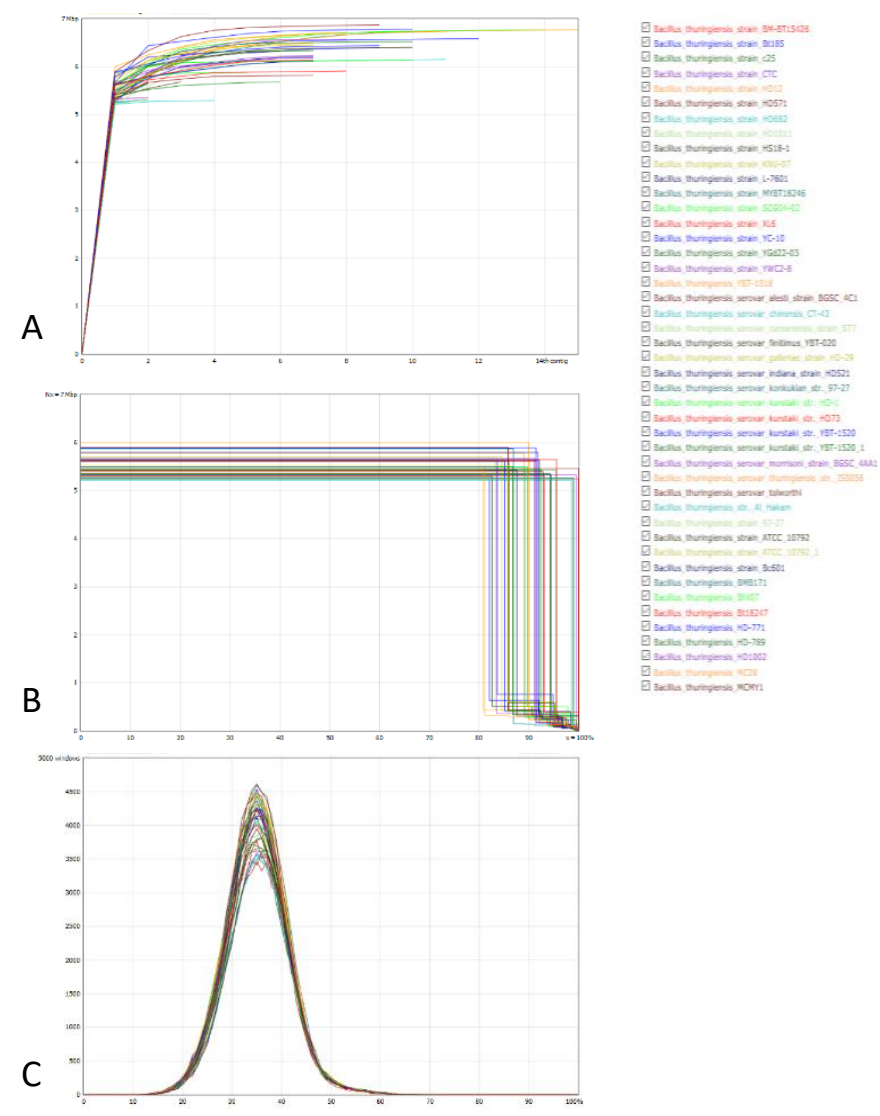

Figure 1. Genomes assembly assessment done by QUAST analysis.

\subsection{Genome status of $B$. thuringiensis}

There are a total of forty-four completed genomes of B. thuringiensis available in NCBI GenBank (as of, 2018). Three strains were found to be having two different complete genome submissions, which are the B. thuringiensis serovar konkukian strain 97-27, B. thuringiensis strain ATCC 10792 and $B$. thuringiensis serovar kurstaki strain YBT-1520. Both completed genomes of all three strains were included in the analysis for comparison purpose. All genomes of B. thuringiensis were inserted into the pipeline of QUAST version 4.6.3 for genome assembly quality assessment. 


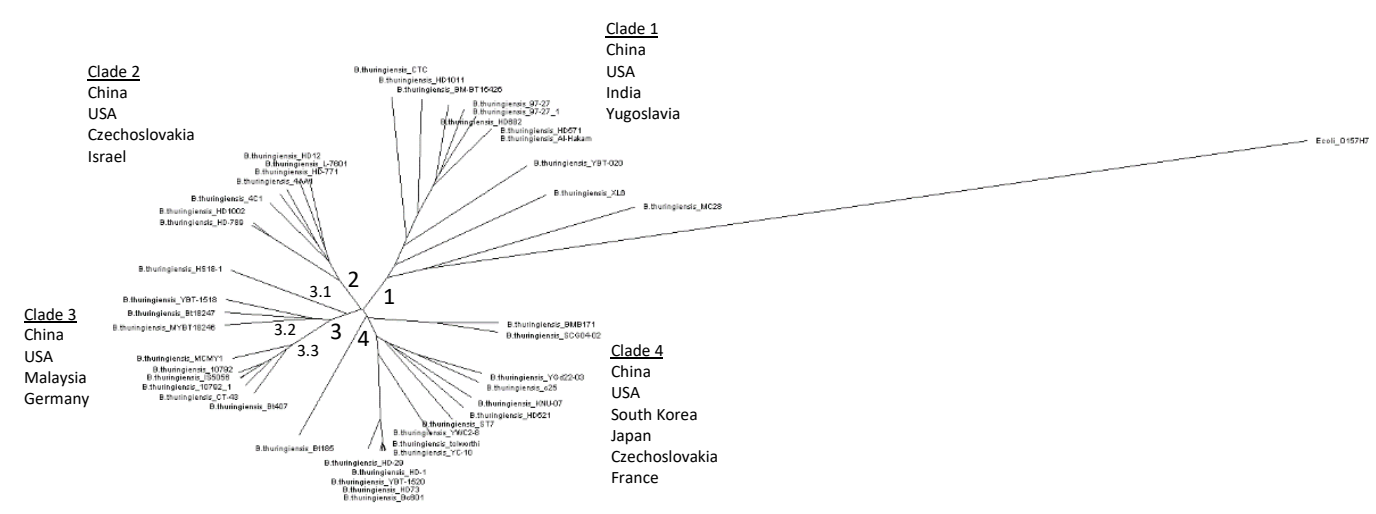

Figure 2. Phylogenetic tree generated for forty-five genomes of $\boldsymbol{B}$. thuringiensis strains and their origin countries according to each clade.

The Results was exported in HTML format and shown in Figure 1. Their genomes size varied between 5.20 (B. thuringiensis strain BM-BT15426) and 6.90 (B. thuringiensis serovar tolworthi) mega base pairs (MB) (Figure 1A). Figure 1B shows the largest contig length, $\mathrm{L}$ accounted for at least $80 \%$ to $100 \%$ of the bases of the assembly and Figure 1C shows the overall GC content of all genomes which was peaked at $35 \%$ approximately.

\subsection{Phylogenetic Information of $B$. thuringiensis}

For phylogenetic reconstruction, B. thuringiensis genomes data were inserted into the Gegenees software version 2.2.1 together with a genome of Escherichia coli strain O157:H7 Sakai as a control for fragmented whole genome alignment and comparison analysis. Escherichia coli strain O157:H7 Sakai was presented with zero percent similarities with all B. thuringiensis strains. The target strain, B. thuringiensis MCMY1 matched the best with $B$. thuringiensis strain $\mathrm{Bt} 407$ and $B$. thuringiensis serovar thuringiensis strain IS5056 at $87 \%$. Both genomes of the same strains, $B$. thuringiensis serovar konkukian strain 97-27, B. thuringiensis strain ATCC 10792 and $B$. thuringiensis serovar kurstaki strain YBT-1520 matched to each other in more than $99 \%$. B. thuringiensis strain MC28 matched the least to all the other genomes at the lowest value of $45 \%$ with $B$. thuringiensis strain XL6. The fragmented whole genome alignment and comparison analysis was exported to SplitsTree software and converted into a phylogram based on the value of phylogenetic similarities. Figure 2 shows the phylogenetic tree generated with four mains clades were observed from the tree. Most of the B. thuringiensis strains were isolated from soil samples and insects body from all around the world, although some origins of the samples were not documented (Table 1). The target B. thuringiensis MCMY1 strain was classified in clade 3 and shared the same branch 3.3 with $B$. thuringiensis serovar thuringiensis strain IS5056, $B$. thuringiensis strain CT-43, B. thuringiensis strain Bt407 and both B. thuringiensis strain ATCC 10792 strains. Other members of Clade 3 are originated from China, USA and Germany. Clade 1 has the longest branches of all, this represents the group has the most diversified members among all.

\subsection{Copper Homeostasis Genes of $B$. thuringiensis}

The genomes data were imported to Rapid Annotations using Subsystems Technology (RAST) for annotations and the genes annotated to the subsystems of copper homeostasis, copper tolerance, copper uptake, copper transport system and blue copper proteins were retrieved from the database. Genes that are present in all genomes are the Coppertranslocating P-type ATPase (CopA/B), Copper resistance protein $\mathrm{C} / \mathrm{D}(\mathrm{CopC} / \mathrm{D})$ and Conserved membrane protein in copper uptake $(\mathrm{YcnI})$. $\mathrm{YcnI}$ is a protein that is speculated to be involving in copper acquisition and it is important during copper limiting conditions [15]. CopA/B is a type of protein that convey copper ions across cell surface and intracellular membrane [16], Cop C/D are the soluble periplasmic chaperone that bind to $\mathrm{Cu}(\mathrm{I})$ and $\mathrm{Cu}(\mathrm{II})$, they are hypothesized to be the transporter of 
essential copper through the inner membrane of cytoplasm [17]. The copper resistant CopABCD were reported from the Pseudomonas syringae pv. tomato genome [18]. Figure 3 shows the genes comparisons by using Blast Ring Image Generator (BRIG), all forty five genomes have shown high similarities in the CopABCD family together with the Coppersensing transcriptional repressor (CsoR) and Copper (I) chaperone Z (CopZ). Only 23 genomes recorded the gene for Cytoplasmic copper homeostasis protein (CutC) and 19 genomes harbored the gene for Magnesium and Cobalt efflux protein (CorC). Figure 3 depicts the diversity of the the CutC and CorC genes in the forty-five $B$. thuringiensis genomes. The target genome $B$. thuringiensis MCMY1 harbors 11 genes except for Magnesium and cobalt efflux protein (CorC). CutC genes have been reported to bind to free copper radicals and reduce stress caused by copper contamination [19] while CorC gene plays a role in the transportation of Magnesium and Cobalt ions which has an indirect function to relieve the oxidative stress that caused by copper and other metal ions [20].

As mentioned in the introductory section, some previous studies have stated that $B$. thuringiensis has been associated a wide range of environments and it can tolerate a different levels of copper induced stress $[4,5,6,7,21,22]$. However the degree of tolerance for copper recorded in their studies are contradistinctive. This can be caused by the unstandardized methodology practiced among the scientists or probably this can be an indicator to show that each $B$. thuringiensis strain that lives in a particular area have adapted to have its own ability to tolerate the copper contamination. Despite the lack of available publications related to the copper tolerance of $B$. thuringiensis in genetic aspect, the findings of current study suggest that Copper resistance genes family, CopABCDZ and its repressor (CsoR) may be the conserved genes in most of the B. thuringiensis genomes. However, the presence and the content of $\mathrm{CutC}$ and $\mathrm{CorC}$ genes in B. thuringiensis genome may play a role in altering the degree of tolerance to copper for each distinctive strain. Further investigations are required in order to support this hypothesis by understanding the expressions of these genes in B. thuringiensis during induced copper stress condition.

\section{Acknowledgements}

The authors are grateful for the financial support rendered by Ministry of Higher Education (Grant no. FRGS0455).

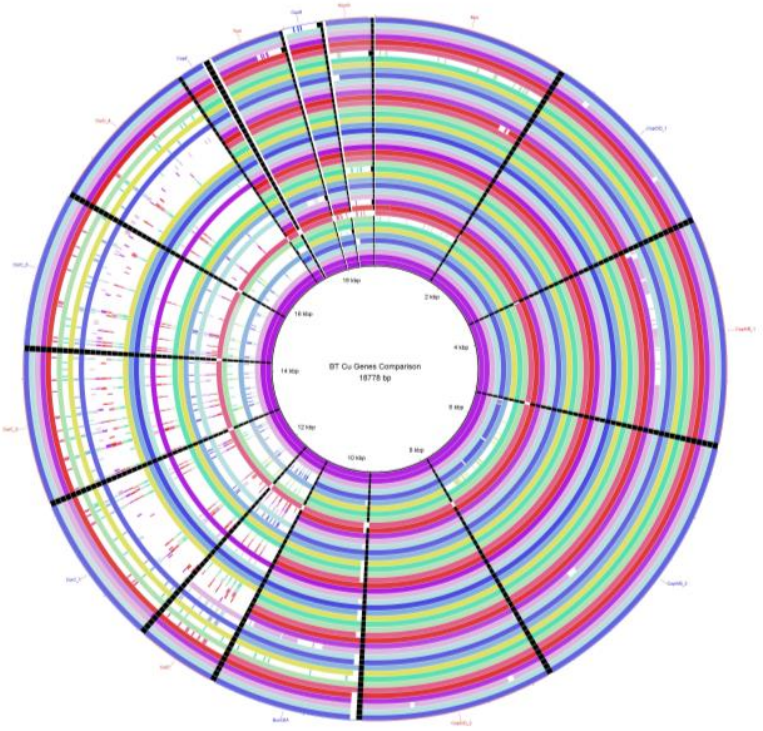

Figure 3. Copper genes comparisons by using Blast Ring Image Generator (BRIG).

\section{References}

[1] Ladomersky, E. and Petris, M. J. (2015). Copper tolerance and virulence in bacteria. Metallomics. 7(6): 957-964.

[2] Bernard, L., Maron, P. A., Mougel, C., Nowak, V., Leveque, J., Marol, C., Balesdent, J., Gibiat, F. and Ranjard, L. (2009). Contamination of soil by copper affects the dynamics, diversity, and activity of soil bacterial communities involved in wheat decomposition and carbon storage. Applied and Environmental Microbiology. 75(23): 7565-7569.

[3] Sansinenea, E. (2012). Discovery and description of Bacillus thuringiensis. In: Sansinenea, E. (Ed). Bacillus thuringiensis Biotechnology. Springer, Dordrecht. http://doi.org/10.1007/978-94-007-30212_1.

[4] Khosravi, V., Ardejani, F. D., Aryafar, A. and Sedighi, M. (2015). Biosorption of metal ions from Sarcheshmeh acid mine drainage by immobilized Bacillus thuringiensis in a fixed-bed column. International Journal of Chemical and Molecular Engineering. 9(11): 1317- 1323.

[5] Marandi, Reza. (2011). Bioextraction of $\mathrm{Cu}$ (II) ions from acid mine drainage by Bacillus thuringiensis. International Journal of Biological Engineering. 1(1):11-17.

[6] Oves, M., Khan, M. S. and Zaidi, A. (2013). Biosorption of heavy metals by Bacillus thuringiensis strain OSM29 originating from industrial effluent contaminated north Indian soil. Saudi Journal of Biological Sciences. 20: 121-129.

[7] Hassen, A., Saidi, N. Cherif, M. and Boudabous, A. (1998). Effects of heavy metals on Pseudomonas 
aeruginosa and Bacillus thuringiensis. Bioresource Technology. 65: 73-82.

[8] Low, Y. Y., Chin, G. J. W. L., Budiman, C., Joseph, C. G., Musta, B. and Rodrigues, K. F. (2018). Adaptive strategies of Bacillus thuringiensis isolated from acid mine drainage site in Sabah, Malaysia. Indian Journal of Microbiology. https://doi.org/10.1007/s12088-017-0701-1.

[9] Gurevich, A., Saveliev, V., Vyahhi, N. and Tesler, G. (2013). QUAST: quality assessment tool for genome assemblies. Genome Analysis. 29(8): 1072-1075.

[10] Agren, J., Sundstrom, A., Hafstrom, T. and Segerman, B. (2012). Gegenees: Fragmented alignment of multiple genomes for determining phylogenomic distances and genetic signatures unique for specified target groups. PLOS ONE. 7(6): e39107.

[11] Hudson, D. H. and Bryant, D. (2006). Application of phylogenetic networks in evolutionary studies. Molecular Biology and Evolution. 23(2): 254-267.

[12] Aziz, R. K., Bartels, D., Best, A. A., Dejongh, M., Disz, T., Edwards, R. A., Formsma, K., Gerdes, S., Glass, E. M., Kubal, M., Meyer, F., Olsen, G. J. Olson, R., Osterman, A. L., Overbeek, R. A., Mcneil, L. K., Paarman, D., Paczian, T., Parrello, B., Pusch, G. D., Reich, C., Stevens, R., Vassieva, O., Vonstein, V.,Wilke, A. and Zagnitko, O. The RAST server: rapid annotations using subsystems technology. BMC Genomics. 9: 75.

[13] Overbeek, R. Olson, R., Puschm G. D., Olsen, G. J., Davis, J. J., Disz, T., Edwards, R. A., Gerdes, S., Parrello, B., Shukla, M., Vonstein, V., Wattam, A. R., Xia, F. and Stevens, R. (2014). The SEED and the rapid annotation of microbial genomes using subsystems technology (RAST). Nucleic Acids Research. 42.

[14] Alikhan, N. F., Petty, N. K., Zakour, N. L. B. and Beatson, S. A. (2011). Blast Ring Image Generator (BRIG): simple prokaryote genome comparisons. BMC Genomics. 12:402. PMID: 21824423.

[15] Chillappagari, S., Miethke, M., Trip, H., Kuipers, O. P. and Marahiel, M. A. (2009). Copper acquisition is mediated by $\mathrm{YcnJ}$ and regulated by $\mathrm{YcnK}$ and CsoR in Bacillus subtilis. Journal of Bacteriology. 191(7): 2362-2370.

[16] Vollmecke, C., Drees, S. L., Reimann, J., Albers, S. and Lubben, M. (2012). The ATPase CopA and CopB both contribute to copper resistance of the thermoacidophilic archaeon Sulfolobus solfataricus. Microbiology. 158:1622-1633.

[17] Bondarczuk, K. and Piotrowska-Seget, Z. (2013). Molecular basis of active copper resistance mechanisms in Gram-negative bacteria. Cell Biology and Toxicology. 29: 397-405.

[18] Lim, C. and Cooksey, D. A. (1993). Characterization of chromosomal homologs of the plasmid-borne copper resistance operon of Pseudomonas syringae. Journal of Bacteriology. 175(14): 4492-4498.

[19] Li, Y., Du, J., Zhang, P. and Ding, J. (2010). Crystal structure of human copper homeostasis protein CutC reveals a potential copper-binding site. Journal of Structural Biology. 169: 399-405.

[20] Nies, D. H. (2003). Efflux-mediated heavy metal resistance in prokaryotes. FEMS Microbiology Reviews. 27:313-339.
[21] Jaafar, R. S., Al-Sulaimi, A. A., Al-Taee, A. M. R. and Aldoghachi, F. J. (2015a). Bioaccumulation of some heavy metals by metal resistant Bacillus thuringiensis isolated from soil in basra governorateIraq. International Journal of Current Research. 7(6): 16709-16713.

[22] Jaafar, R., Al-Sulaimi, A., Al-Taee, A. and Aldoghachi, F. (2015b). Biosorption of some heavy metals by metal resistant Bacillus thuringiensis isolated from soil in Basra Governorate-Iraq. Journal of Biology, Agriculture and Healthcare. 5(10). 